\title{
The Fundamental Theorem of Asset Pricing
}

The subsequent theorem is one of the pillars supporting the modern theory of Mathematical Finance.

\section{Fundamental Theorem of Asset Pricing:}

The following two statements are essentially equivalent for a model $S$ of a financial market:

(i) $S$ does not allow for arbitrage (NA)

(ii) There exists a probability measure $Q$ on the underlying probability space $(\Omega, \mathcal{F}, \mathbb{P})$, which is equivalent to $\mathbb{P}$ and under which the process is a martingale (EMM).

We have formulated this theorem in vague terms which will be made precise in the sequel: we shall formulate versions of this theorem below which use precise definitions and avoid the use of the word essentially above. In fact, the challenge is precisely to turn this vague "meta-theorem" into sharp mathematical results.

The story of this theorem started - like most of modern Mathematical Finance - with the work of F. Black, M. Scholes [3] and R. Merton [25]. These authors consider a model $S=\left(S_{t}\right)_{0 \leq t \leq T}$ of geometric Brownian motion proposed by P. Samuelson [30], which today is widely known under the name of Black-Scholes model. Presumably every reader of this article is familiar with the by now wellknown technique to price options in this framework (compare eqf04/003: Risk Neutral Pricing): one changes the underlying measure $\mathbb{P}$ to an equivalent measure $Q$ under which the discounted stock price process is a martingale. Subsequently one prices options (and other derivatives) by simply taking expectations with respect to this "risk neutral" or "martingale" measure $Q$.

In fact, this technique was not the novel feature of [3] and [25]. It was used by actuaries for some centuries and it was also used by L. Bachelier [2] in 1900 who considered Brownian motion (which, of course, is a martingale) as a model $S=\left(S_{t}\right)_{0 \leq t \leq T}$ of a stock price process. In fact, the prices obtained by Bachelier by this method were - at least for the empirical data considered by Bachelier himself - very close to those derived from the celebrated BlackMerton-Scholes formula (compare [34]).

The decisive novel feature of the Black-Merton-Scholes approach was the argument which links this pricing technique with the notion of arbitrage: the pay-off function of an option can be precisely replicated by trading dynamically in the underlying stock. This idea, which is credited in footnote 3 of 
[3] to R. Merton, opened a completely new perspective on how to deal with options, as it linked the pricing issue with the idea of hedging, i.e., dynamically trading in the underlying asset.

The technique of replicating an option is completely absent in Bachelier's early work; apparently the idea of "spanning" a market by forming linear combinations of primitive assets first appears in the Economics literature in the classic paper by K. Arrow [1]. The mathematically delightful situation, that the market is complete in the sense that all derivatives can be replicated, occurs in the Black-Scholes model as well as in Bachelier's original model of Brownian motion (compare eqf04/008: Second Fundamental Asset Pricing Theorem). Another example of a model in continuous time sharing this property is the compensated Poisson process, as observed by J. Cox and S. Ross [4]. Roughly speaking, these are the only models in continuous time sharing this seducingly beautiful "martingale representation property" (see [16] and [39] for a precise statement on the uniqueness of these families of models).

Appealing as it might be, the consideration of "complete markets" as above is somewhat dangerous from an economic point of view: the precise replicability of options, which is a sound mathematical theorem in the framework of the above models, may lead to the illusion that this is also true in economic reality. But, of course, these models are far from matching reality in a one-to-one manner. Rather they only highlight important aspects of reality; therefore they should not be considered as ubiquitously appropriate.

For many purposes it is of crucial importance to put oneself into a more general modeling framework.

When the merits as well as the limitations of the Black-Merton-Scholes approach unfolded in the late 70's, the investigations on the Fundamental Theorem of Asset Pricing started. As J. Harrison and S. Pliska formulate it in their classic paper [15]: "it was a desire to better understand their formula which originally motivated our study,..."

The challenge was to obtain a deeper insight into the relation of the following two aspects: on the one side the methodology of pricing by taking expectations with respect to a properly chosen "risk neutral" or "martingale" measure $Q$; on the other hand the methodology of pricing by "no arbitrage" considerations. Why, after all, do these two seemingly unrelated approaches yield identical results in the Black-Merton-Scholes approach? Maybe even more importantly: how far can this phenomenon be extended to more involved models?

To the best of my knowledge the first one to take up these questions in a systematic way was S. Ross ([29]; see also [4], [28], and [27]). 
He chose the following setting to formalize the situation: fix a topological, ordered vector space $(X, \tau)$, modeling the possible cash flows (e.g. the pay-off function of an option) at a fixed time horizon $T$. A good choice is, e.g. $X=L^{p}(\Omega, \mathcal{F}, \mathbb{P})$, where $1 \leq p \leq \infty$ and $\left(\Omega, \mathcal{F},\left(\mathcal{F}_{t}\right)_{0 \leq t \leq T}, \mathbb{P}\right)$ is the underlying filtered probability space. The set of marketed assets $M$ is a subspace of $X$.

In the context of a stock price process $S=\left(S_{t}\right)_{0 \leq t \leq T}$ as above, one might think of $M$ as all the outcomes of an initial investment $x \in \mathbb{R}$ plus the result of subsequent trading according to a predictable trading strategy $H=\left(H_{t}\right)_{0 \leq t \leq T}$. This yields (in discounted terms) an element

$$
m=x+\int_{0}^{T} H_{t} d S_{t}
$$

in the set $M$ of marketed claims. It is natural to price the above claim $m$ by setting $\pi(m)=x$, as this is the net investment necessary to finance the above claim $m$.

For notational convenience we shall assume in the sequel that $S$ is a onedimensional process. It is straightforward to generalize to the case of $d$ risky assets by assuming that $S$ is $\mathbb{R}^{d}$-valued and replacing the above integral by

$$
m=x+\int_{0}^{T} \sum_{i=1}^{d} H_{t}^{i} d S_{t}^{i} .
$$

Some words of warning about the stochastic integral (1) seem necessary. The precise admissibility conditions which should be imposed on the stochastic integral (1), in order to make sense both mathematically as well as economically, are a subtle issue. Much of the early literature on the Fundamental Theorem of Asset Pricing struggled exactly with this question. An excellent reference is [14]. In [29] S. Ross circumvented this problem by deliberately leaving this issue aside and simply starting with the modeling assumption that the subset $M \subseteq X$ as well as a pricing operator $\pi: M \rightarrow \mathbb{R}$ are given.

Let us now formalize the notion of arbitrage. In the above setting, we say that the no arbitrage assumption is satisfied if, for $m \in M$, satisfying $m \geq 0, \mathbb{P}$-a.s. and $\mathbb{P}[m>0]>0$, we have $\pi(m)>0$. In prose this means that it is not possible to find a claim $m \in M$, which bears no risk (as $m \geq 0, \mathbb{P}_{\text {- }}$ a.s.), yields some gain with strictly positive probability (as $\mathbb{P}[m>0]>0$ ), and such that its price $\pi(m)$ is less than or equal to zero.

The question now arises whether it is possible to extend $\pi: M \rightarrow \mathbb{R}$ to a non-negative, continuous linear functional $\pi^{*}: X \rightarrow \mathbb{R}$.

What does this have to do with the issue of martingale measures? This 
theme was developed in detail by M. Harrison and D. Kreps [14]. Suppose that $X=L^{p}(\Omega, \mathcal{F}, \mathbb{P})$ for some $1 \leq p<\infty$, that the price process $S=\left(S_{t}\right)_{0 \leq t \leq T}$ satisfies $S_{t} \in X$, for each $0 \leq t \leq T$, and that $M$ contains (at least) the "simple integrals" on the process $S=\left(S_{t}\right)_{0 \leq t \leq T}$ of the form

$$
m=x+\sum_{i=1}^{n} H_{i}\left(S_{t_{i}}-S_{t_{i-1}}\right) .
$$

Here $x \in \mathbb{R}, \quad 0=t_{0}<t_{1}<\cdots<t_{n}=T$ and $\left(H_{i}\right)_{i=1}^{n}$ is a (say) bounded process which is predictable, i.e. $H_{i}$ is $\mathcal{F}_{t_{i-1}}$-measurable. The sums in (2) are the Riemann sums corresponding to the stochastic integrals (1). The Riemann sums (2) have a clear-cut economic interpretation (see [14]). In (2) we do not have to bother about subtle convergence issues as only finite sums are involved in the definition. It therefore is a traditional (minimal) requirement that the Riemann sums of the form (2) are in the space $M$ of marketed claims; naturally, the price of a claim $m$ of the form (2) should be defined as $\pi(m)=x$.

Now suppose that the functional $\pi$, which is defined for the claims of the form (2) can be extended to a continuous, non-negative functional $\pi^{*}$ defined on $X=L^{p}(\Omega, \mathcal{F}, \mathbb{P})$. If such an extension $\pi^{*}$ exists, it is induced by some function $g \in L^{q}(\Omega, \mathcal{F}, \mathbb{P})$, where $\frac{1}{p}+\frac{1}{q}=1$. The non-negativity of $\pi^{*}$ is tantamount to $g \geq 0, \mathbb{P}$-a.s., and the fact that $\pi^{*}(1)=1$ shows that $g$ is the density of a probability measure $Q$ with Radon-Nikodym derivative $\frac{d Q}{d \mathbb{P}}=g$.

If we can find such an extension $\pi^{*}$ of $\pi$, we thus find a probability measure $Q$ on $(\Omega, \mathcal{F}, \mathbb{P})$ for which

$$
\pi^{*}\left(\sum_{i=1}^{n} H_{i}\left(S_{t_{i}}-S_{t_{i-1}}\right)\right)=\mathbb{E}_{Q}\left[\sum_{i=1}^{n} H_{i}\left(S_{t_{i}}-S_{t_{i-1}}\right)\right]
$$

for every bounded predictable process $H=\left(H_{i}\right)_{i=1}^{n}$ as above, which is tantamount to $\left(S_{t}\right)_{0 \leq t \leq T}$ being a martingale (see [14, Th. 2], or [11, Lemma 2.2.6]).

Summing up: in the case $1 \leq p<\infty$, finding a continuous, non-negative extension $\pi^{*}: L^{p}(\Omega, \mathcal{F}, \mathbb{P}) \rightarrow \mathbb{R}$ of $\pi$ amounts to finding a $\mathbb{P}$-absolutely continuous measure $Q$ with $\frac{d Q}{d \mathbb{P}} \in L^{q}$ and such that $\left(S_{t}\right)_{0 \leq t \leq T}$ is a martingale under $Q$.

At this stage it becomes clear that in order to find such an extension $\pi^{*}$ of $\pi$, the Hahn-Banach theorem should come into play in some form, e.g., in one of the versions of the separating hyperplane theorem.

In order to be able to do so, S. Ross assumes ([29, p. 472]) that "...we will endow $X$ with a strong enough topology to insure that the positive orthant $\{x \in X \mid x>0\}$ is an open set,...". In practice, the only infinite-dimensional 
ordered topological vector space $X$, such that the positive orthant has nonempty interior, is $X=L^{\infty}(\Omega, \mathcal{F}, \mathbb{P})$, endowed with the topology induced by $\|\cdot\|_{\infty}$.

Hence the two important cases, applying to S. Ross' hypothesis, are when either the probability space $\Omega$ is finite, so that $X=L^{p}(\Omega, \mathcal{F}, \mathbb{P})$ simply is finite dimensional and its topology does not depend on $1 \leq p \leq \infty$, or if $(\Omega, \mathcal{F}, \mathbb{P})$ is infinite and $X=L^{\infty}(\Omega, \mathcal{F}, \mathbb{P})$ equipped with the norm $\|\cdot\|_{\infty}$.

After these preparations we can identify the two convex sets to be separated: let $A=\{m \in M: \pi(m) \leq 0\}$ and $B$ be the interior of the positive cone of $X$. Now make the easy, but crucial, observation: these sets are disjoint if and only if the no arbitrage condition is satisfied. As one always can separate an open convex set from a disjoint convex set, we find a functional $\tilde{\pi}$ which is strictly positive on $B$, while $\tilde{\pi}$ takes non-positive values on $A$. By normalizing $\tilde{\pi}$, i.e., letting $\pi^{*}=\tilde{\pi}(1)^{-1} \tilde{\pi}$ we have thus found the desired extension.

In summary, the first precise version of the Fundamental Theorem of Asset Pricing is established in [29], the proof relying on the Hahn-Banach theorem. There are, however, serious limitations: in the case of infinite $(\Omega, \mathcal{F}, \mathbb{P})$ the present result only applies to $L^{\infty}(\Omega, \mathcal{F}, \mathbb{P})$ endowed with the norm topology. In this case the continuous linear functional $\pi^{*}$ only is in $L^{\infty}(\Omega, \mathcal{F}, \mathbb{P})^{*}$ and not necessarily in $L^{1}(\Omega, \mathcal{F}, \mathbb{P})$; in other words we cannot be sure that $\pi^{*}$ is induced by a probability measure $Q$, as it may happen that $\pi^{*} \in L^{\infty}(\Omega, \mathcal{F}, \mathbb{P})^{*}$ also has a singular part.

Another drawback, which already appears in the case of finite-dimensional $\Omega$ (in which case $\pi^{*}$ certainly is induced by some $Q$ with $\frac{d Q}{d \mathbb{P}}=g \in L^{1}(\Omega, \mathcal{F}, \mathbb{P})$ ) is the following: we cannot be sure that the function $g$ is strictly positive $\mathbb{P}$ a.s. or, in other words, that $Q$ is equivalent to $\mathbb{P}$.

After this early work by S. Ross a major advance in the theory was achieved between '79 and '81 by three seminal papers [14], [15], [24] by M. Harrison, D. Kreps and S. Pliska. In particular, [14] is a landmark in the field. It uses a similar setting as [29], namely an ordered topological vector space $(X, \tau)$ and a linear functional $\pi: M \rightarrow \mathbb{R}$, where $M$ is a linear subspace of $X$. Again the question is whether there exists an extension of $\pi$ to a linear, continuous, strictly positive $\pi^{*}: X \rightarrow \mathbb{R}$. This question is related in [14] to the issue whether $(M, \pi)$ is viable as a model of economic equilibrium. Under proper assumptions on the convexity and continuity of the preferences of agents this is shown to be equivalent to the extension discussed above.

The paper [14] also analyses the case when $\Omega$ is finite. Of course, only processes $S=\left(S_{t}\right)_{t=0}^{T}$ indexed by finite, discrete time $\{0,1, \ldots, T\}$ make sense 
in this case. For this easier setting the following precise theorem was stated and proved in the subsequent paper [15] by J. Harrison and S. Pliska:

Theorem 1. ([15, Th. 2. 7.]): Suppose the stochastic process $S=\left(S_{t}\right)_{t=0}^{T}$ is based on a finite, filtered, probability space $\left(\Omega, \mathcal{F},\left(\mathcal{F}_{t}\right)_{t=0}^{T}, \mathbb{P}\right)$. The market model contains no arbitrage possibilities if and only if there is an equivalent martingale measure for $S$.

The proof again relies on a (finite-dimensional version) of the HahnBanach theorem plus an extra argument making sure to find a measure $Q$ which is equivalent to $\mathbb{P}$. M. Harrison and S. Pliska thus have achieved a precise version of the above meta-theorem in terms of equivalent martingale measures which does not use the word "essentially". Actually, the theme of the Harrison-Pliska theorem goes back much further, to the work of A. Shimony [35] and J. Kemeny [22] on symbolic logic in the tradition of R. Carnap, B. de Finetti, and F. Ramsey. These authors showed that, in a setting with only finitely many states of the world, a family of possible bets does not allow (by taking linear combinations) for making a riskless profit (i.e. one certainly does not lose but wins with strictly positive probability), if and only if there is a probability measure $Q$ on these finitely many states, which prices the possible bets by taking conditional $Q$-expectations.

The restriction to finite $\Omega$ is very severe in applications: the flavor of the theory, building on Black-Scholes-Merton, is precisely the concept of continuous time. Of course, this involves infinite probability spaces $(\Omega, \mathcal{F}, \mathbb{P})$.

Many interesting questions were formulated in the papers [14] and [15] hinting on the difficulties to prove a version of the Fundamental Theorem of Asset Pricing beyond the setting of finite probability spaces.

A major break-through in this direction was achieved by D. Kreps [24]: as above, let $M \subseteq X$ and a linear functional $\pi: M \rightarrow \mathbb{R}$ be given. The typical choice for $X$ will now be $X=L^{p}(\Omega, \mathcal{F}, \mathbb{P})$, for $1 \leq p \leq \infty$, equipped with the topology $\tau$ of convergence in norm, or, if $X=L^{\infty}(\Omega, \mathcal{F}, \mathbb{P})$, equipped with the Mackey topology $\tau$ induced by $L^{1}(\Omega, \mathcal{F}, \mathbb{P})$. This setting will make sure that a continuous linear functional on $(X, \tau)$ will be induced by a measure $Q$ which is absolutely continuous with respect to $\mathbb{P}$.

The no arbitrage assumption means that $M_{0}:=\{m \in M: \pi(m)=0\}$ intersects the positive orthant $X_{+}$of $X$ only in $\{0\}$. In order to obtain an extension of $\pi$ to a continuous, linear functional $\pi^{*}: X \rightarrow \mathbb{R}$ we have to find an element in $(X, \tau)^{*}$ which separates the convex set $M_{0}$ from the disjoint convex set $X_{+} \backslash\{0\}$, i.e., the positive orthant of $X$ with 0 deleted.

Easy examples show that, in general, this is not possible. In fact, this is not much of a surprise (if $X$ is infinite-dimensional) as we know that some 
topological condition is needed for the Hahn-Banach theorem to work.

It is always possible to separate a closed convex set from a disjoint compact convex set by a continuous linear functional. In fact, one may even get strict separation in this case. It is this version of the Hahn-Banach theorem which D. Kreps eventually applies.

But how? After all, neither $M_{0}$ nor $X_{+} \backslash\{0\}$ are closed in $(X, \tau)$, let alone compact.

Here is the ingenious construction of D. Kreps: define

$$
A=\overline{M_{0}-X_{+}},
$$

where the bar denotes the closure with respect to the topology $\tau$. We shall require that $A$ still satisfies

$$
A \cap X_{+}=\{0\}
$$

This property is baptized as "no free lunch" by D. Kreps:

Definition 2. [24]: The financial market defined by $(X, \tau), M$, and $\pi$ admits a free lunch if there are nets $\left(m_{\alpha}\right)_{\alpha \in I} \in M_{0}$ and $\left(h_{\alpha}\right)_{\alpha \in I} \in X_{+}$such that

$$
\lim _{\alpha \in I}\left(m_{\alpha}-h_{\alpha}\right)=x
$$

for some $x \in X_{+} \backslash\{0\}$.

It is easy to verify that the negation of the above definition is tantamount to the validity of (4).

The economic interpretation of the "no free lunch" condition is a sharpening of the "no arbitrage condition". If the latter is violated, we can simply find an element $x \in X_{+} \backslash\{0\}$ which also lies in $M_{0}$. If the former fails, we cannot quite guarantee this, but we can find $x \in X_{+} \backslash\{0\}$ which can be approximated in the $\tau$-topology by elements of the form $m_{\alpha}-h_{\alpha}$. The passage from $m_{\alpha}$ to $m_{\alpha}-h_{\alpha}$ means that agents are allowed to "throw away money", i.e. to abandon a positive element $h_{\alpha} \in X_{+}$. This combination of the "free disposal" assumption with the possibility of passing to limits is crucial in Kreps' approach (3) as well as in most of the subsequent literature. It was shown in [32, Ex. 3.3]; (compare also [33]) that the (seemingly ridiculous) "free disposal" assumption cannot be dropped.

Definition (3) is tailor-made for the application of Hahn-Banach. If the no free lunch condition (4) is satisfied, we may, for any $h \in X_{+}$, separate the $\tau$-closed, convex set $A$ from the one-point set $\{h\}$ by an element $\pi_{h} \in(X, \tau)^{*}$. As $0 \in A$ we may assume that $\left.\pi_{h}\right|_{A} \leq 0$ while $\pi_{h}(h)>0$. We thus have obtained a non-negative (as $-X_{+} \subseteq A$ ), continuous linear functional $\pi_{h}$ which 
is strictly positive on a given $h \in X_{+}$. Supposing that $X_{+}$is $\tau$-separable (which is the case in the above setting of $L^{p}$-spaces if $(\Omega, \mathcal{F}, \mathbb{P})$ is countably generated), fix a dense sequence $\left(h_{n}\right)_{n=1}^{\infty}$ and find strictly positive scalars $\mu_{n}>0$ such that $\pi^{*}=\sum_{n=1}^{\infty} \mu_{n} \pi_{h_{n}}$ converges to a probability measure in $(X, \tau)^{*}=L^{q}(\Omega, \mathcal{F}, \mathbb{P})$, where $\frac{1}{p}+\frac{1}{q}=1$. This yields the desired extension $\pi^{*}$ of $\pi$ which is strictly positive on $X_{+} \backslash\{0\}$.

We still have to specify the choice of $\left(M_{0}, \pi\right)$. The most basic choice is to take for given $S=\left(S_{t}\right)_{0 \leq t \leq T}$ the space generated by the "simple integrands" (2) as proposed by J. Harrison and D. Kreps [14]. We thus may deduce from Kreps' arguments in [24] the following version of the Fundamental Theorem of Asset pricing.

Theorem 3. Let $(\Omega, \mathcal{F}, \mathbb{P})$ be countably generated and $X=L^{p}(\Omega, \mathcal{F}, \mathbb{P})$ endowed with the norm topology $\tau$, if $1 \leq p<\infty$, or the Mackey topology induced by $L^{1}(\Omega, \mathcal{F}, \mathbb{P})$, if $p=\infty$.

Let $S=\left(S_{t}\right)_{0 \leq t \leq T}$ be a stochastic process taking values in $X$. Define $M_{0} \subseteq X$ to consist of the simple stochastic integrals $\sum_{i=1}^{n} H_{i}\left(S_{t_{i}}-S_{t_{i-1}}\right)$ as in (2).

Then the "no free lunch" condition (3) is satisfied if and only if there is a probability measure $Q$ with $\frac{d Q}{d \mathbb{P}} \in L^{q}(\Omega, \mathcal{F}, \mathbb{P})$, where $\frac{1}{p}+\frac{1}{q}=1$, such that $\left(S_{t}\right)_{0 \leq t \leq T}$ is a $Q$-martingale.

This remarkable theorem of D. Kreps sets new standards. For the first time, we have a mathematically precise statement of our meta-theorem applying to a general class of models in continuous time. There are still some limitations, however.

When applying the theorem to the case $1 \leq p<\infty$ we find the requirement $\frac{d Q}{d \mathbb{P}} \in L^{q}(\Omega, \mathcal{F}, \mathbb{P})$ for some $q>1$, which is not very pleasant. After all, we want to know: what exactly corresponds (in terms of some no arbitrage condition) to the existence of an equivalent martingale measure $Q$ ? The $q$-moment condition is unnatural in most applications. In particular, it is not invariant under equivalent changes of measures as is done often in the applications.

The most interesting case of the above theorem is $p=\infty$. But in this case the requirement $S_{t} \in X=L^{\infty}(\Omega, \mathcal{F}, \mathbb{P})$ is unduly strong for most applications. In addition, for $p=\infty$ we run into the subtleties of the Mackey topology $\tau$ (or the weak-star topology, which does not make much of a difference) on $L^{\infty}(\Omega, \mathcal{F}, \mathbb{P})$. We shall discuss this issue below.

The "heroic period" of the development of the Fundamental Theorem of Asset Pricing marked by S. Ross [29], Harrison-Kreps [14], Harrison-Pliska [15] and D. Kreps [24], put the issue on safe mathematical grounds and 
brought some spectacular results. However, it still left many questions open; quite a number of them were explicitly stated as open problems in these papers.

Subsequently a rather extensive literature developed, answering these problems and opening new perspectives. We cannot give a full account on all of this literature and refer, e.g., to the monograph [11] for more extensive information. We can give an outline.

As regards the situation for $1 \leq p \leq \infty$ in Kreps' theorem, this issue was further developed by D. Duffie and C.F. Huang [12] and, in particular, by C. Stricker [36]. This author related the no free lunch condition of D. Kreps to a theorem by J.A. Yan [37] obtained in the context of the Bichteler-Dellacherie theorem on the characterisation of semi-martingales. Using Yan's theorem, Stricker gave a different proof of Kreps' theorem which does not need the assumption that $(\Omega, \mathcal{F}, \mathbb{P})$ is countably generated.

A beautiful extension of the Harrison-Pliska theorem was obtained in 1990 by R. Dalang, A. Morton and W. Willinger [5]. They showed that, for an $\mathbb{R}^{d}$-valued process $\left(S_{t}\right)_{t=0}^{T}$ in finite discrete time, the no arbitrage condition is indeed equivalent to the existence of an equivalent martingale measure. The proof is surprisingly tricky, at least for the case $d \geq 2$. It is based on the measurable selection theorem (the suggestion to use this theorem is acknowledged to F. Delbaen). Different proofs of the Dalang-Morton-Willinger theorem have been given in [31], [20], [26], [17], and [21].

A important question left unanswered by D. Kreps was whether one can, in general, replace the use of nets $\left(m_{\alpha}-h_{\alpha}\right)_{\alpha \in I}$, indexed by $\alpha$ ranging in a general ordered set $I$, simply by sequences $\left(m_{n}-h_{n}\right)_{n=1}^{\infty}$. In the context of continuous processes $S=\left(S_{t}\right)_{0 \leq t \leq T}$ a positive answer was given by F. Delbaen in [6], if one is willing to make the harmless modification to replace the deterministic times $0=t_{0} \leq t_{1} \leq \cdots \leq t_{n}=T$ in (2) by stopping times $0=\tau_{0} \leq \tau_{1} \leq \cdots \leq \tau_{n}=T$. A second case, where the answer to this question is positive are processes $S=\left(S_{t}\right)_{t=0}^{\infty}$ in infinite, discrete time as shown in $[32]$.

The Banach-Steinhaus theorem implies that, for a sequence $\left(m_{n}-h_{n}\right)_{n=1}^{\infty}$ converging in $L^{\infty}(\Omega, \mathcal{F}, \mathbb{P})$ with respect to the weak-star (or Mackey) topology, the norms $\left(\left\|m_{n}-h_{n}\right\|_{\infty}\right)_{n=1}^{\infty}$ remain bounded ("uniform boundedness principle"). Therefore it follows that in the above two cases of continuous processes $S=\left(S_{t}\right)_{0 \leq t \leq T}$ or processes $\left(S_{t}\right)_{t=0}^{\infty}$ in infinite, discrete time, the "no free lunch" condition of D. Kreps can be equivalently replaced by the "no free lunch with bounded risk" condition introduced in [32]: in (5) above we additionally impose that $\left(\left\|m_{\alpha}-h_{\alpha}\right\|_{\infty}\right)_{\alpha \in I}$ remains bounded. In this case we have that there is a constant $M>0$ such that $m_{\alpha} \geq-M, \mathbb{P}$-a.s. for each 
$\alpha \in I$, which explains the wording "bounded risk".

However, in the context of general semi-martingale models $S=\left(S_{t}\right)_{0 \leq t \leq T}$, a counter-example was given by F. Delbaen and the present author in ([7, Ex. 7.8]) showing that the "no free lunch with bounded risk" condition does not imply the existence of an equivalent martingale measure. Hence, in a general setting and by only using simple integrals, there is no hope to get any more precise information on the free lunch condition than the one provided by Kreps' theorem.

At this stage it became clear that, in order to obtain sharper results, one has to go beyond the framework of simple integrals (2) and rather use general stochastic integrals (1). After all, the simple integrals only are a technical gimmick, analogous to step functions in measure theory. In virtually all the applications, e.g., the replication strategy of an option in the Black-Scholes model, one uses general integrals of the form (1).

General integrands pose a number of questions to be settled. First of all, the integral (1) has to be mathematically well-defined. The theory of stochastic calculus starting with K. Itô, and developed in particular by the Strasbourg school of probability around P.-A. Meyer, provides very precise information on this issue: there is a good integration theory for a given stochastic process $S=\left(S_{t}\right)_{0 \leq t \leq T}$ if and only if $S$ is a semi-martingale (theorem of Bichteler-Dellacherie).

Hence mathematical arguments lead to the model assumption that $S$ has to be a semi-martingale. But what about an economic justification of this assumption? Fortunately the economic reasoning hints in the same direction. It was shown by F. Delbaen and the present author that, for a locally bounded stochastic process $S=\left(S_{t}\right)_{0 \leq t \leq T}$, a very weak form of Kreps' no free lunch condition involving simple integrands (2), implies already that $S$ is a semi-martingale (see [7, Theorem 7.2], for a precise statement).

Hence it is natural to assume that the model $S=\left(S_{t}\right)_{0 \leq t \leq T}$ of stock prices is a semi-martingale so that the stochastic integral (2) makes sense mathematically, for all $S$-integrable, predictable processes $H=\left(H_{t}\right)_{0 \leq t \leq T}$. As pointed out, [14] and [15] impose in addition an admissibility condition to rule out doubling strategies and similar schemes.

Definition 4. ([7, Def. 2.7]): An S-integrable predictable process $H=$ $\left(H_{t}\right)_{0 \leq t \leq T}$ is called admissible if there is a constant $M>0$ such that

$$
\int_{0}^{t} H_{u} d S_{u} \geq-M, \quad \text { a.s., for } 0 \leq t \leq T \text {. }
$$


The economic interpretation is that the economic agent, trading according to the strategy, has to respect a finite credit line $M$.

Let us now sketch the approach of [7]. Define

$$
K=\left\{\int_{0}^{T} H_{t} d S_{t}: H \text { admissible }\right\}
$$

which is a set of (equivalence classes of) random variables. Note that by (6) the elements $f \in K$ are uniformly bounded from below, i.e., $f \geq-M$ for some $M \geq 0$. On the other hand, there is no reason why the positive part $f_{+}$should obey any boundedness or integrability assumption.

As a next step we "allow agents to throw away money" similarly as in Kreps' work [24]. Define

$$
\begin{aligned}
C & =\left\{g \in L^{\infty}(\Omega, \mathcal{F}, \mathbb{P}): g \leq f \text { for some } f \in K\right\} \\
& =\left[K-L_{+}^{0}(\Omega, \mathcal{F}, \mathbb{P})\right] \cap L^{\infty}(\Omega, \mathcal{F}, \mathbb{P}),
\end{aligned}
$$

where $L_{+}^{0}(\Omega, \mathcal{F}, \mathbb{P})$ denotes the set of non-negative measurable functions.

By construction, $C$ consists of bounded random variables, so that we can use the functional analytic duality theory between $L^{\infty}$ and $L^{1}$. The difference of the subsequent definition to Kreps' approach is that it pertains to the norm topology $\|\cdot\|_{\infty}$ rather than to the Mackey topology on $L^{\infty}(\Omega, \mathcal{F}, \mathbb{P})$.

Definition 5. ([11, 2.8]): A locally bounded semi-martingale $S=\left(S_{t}\right)_{0 \leq t \leq T}$ satisfies the no free lunch with vanishing risk condition if

$$
\bar{C} \cap L_{+}^{\infty}(\Omega, \mathcal{F}, \mathbb{P})=\{0\},
$$

where $\bar{C}$ denotes the $\|\cdot\|_{\infty}$-closure of $C$.

Here is the translation of (9) into prose: the process $S$ fails the above condition iff there is a function $g \in L_{+}^{\infty}(\Omega, \mathcal{F}, \mathbb{P})$ with $\mathbb{P}[g>0]>0$ and a sequence $\left(f^{n}\right)_{n=1}^{\infty}$ of the form

$$
f^{n}=\int_{0}^{T} H_{t}^{n} d S_{t}
$$

where $H^{n}$ are admissible integrands, such that

$$
f_{n} \geq g-\frac{1}{n}, \quad \text { a.s. }
$$

Hence the condition of no free lunch with vanishing risk is intermediate between the (stronger) no free lunch condition of D. Kreps and the (weaker) 
no arbitrage condition. The latter would require that there is a non-negative function $g$ with $\mathbb{P}[g>0]>0$ which is of the form

$$
g=\int_{0}^{T} H_{t} d S_{t}
$$

for an admissible integrand $H$. Condition (10) does not quite guarantee this, but something - at least from an economic point of view - very close: we can uniformly approximate from below such a $g$ by the outcomes $f_{n}$ of admissible trading strategies.

The main result of F. Delbaen and the author [7] reads as follows.

Theorem 6. ([7, Corr. 1.2]): Let $S=\left(S_{t}\right)_{0 \leq t \leq T}$ be a locally bounded realvalued semi-martingale.

There is a probability measure $Q$ on $(\Omega, \mathcal{F})$ which is equivalent to $\mathbb{P}$ and under which $S$ is a local martingale if and only if $S$ satisfies the condition of no free lunch with vanishing risk.

This is a mathematically precise theorem which, in my opinion, is quite close to the vague "meta-theorem" at the beginning of this article. The difference to the intuitive "no arbitrage" idea is that the agent has to be willing to sacrifice (at most) the quantity $\frac{1}{n}$ in (10), where we may interpret $\frac{1}{n}$ as, say, 1 Cent.

The proof of the above theorem is rather longish and technical and a more detailed discussion goes beyond the scope of the present article. To the best of my knowledge, no essential simplification of this proof has been achieved so far (compare [19]).

Mathematically speaking, the statement of the theorem looks very suspicious at first glance: after all, the no free lunch with vanishing risk condition pertains to the norm topology of $L^{\infty}(\Omega, \mathcal{F}, \mathbb{P})$. Hence it seems that, when applying the Hahn-Banach theorem, one can only obtain a linear functional in $L^{\infty}(\Omega, \mathcal{F}, \mathbb{P})^{*}$, which is not necessarily of the form $\frac{d Q}{d \mathbb{P}} \in L^{1}(\Omega, \mathcal{F}, \mathbb{P})$, as we have seen in Ross' work [29].

The reason why the above theorem nevertheless is true is a little miracle: it turns out ([7, Th. 4.2]) that, under the assumption of no free lunch with vanishing risk, the set $C$ defined in (8) is automatically weak-star closed in $L^{\infty}(\Omega, \mathcal{F}, \mathbb{P})$. This pleasant fact is not only a crucial step in the proof of the above theorem; maybe even more importantly, it also found other applications. For example, to find general existence results in the theory of utility optimisation (eqf04/009: Expected utility maximization) it is of crucial importance to have a closedness property of the set over which one optimizes: for these applications the above result is very useful (see, e.g., 
[23]).

Without going into the details of the proof let me point out the importance of certain elements in the set $K$. The admissibility rules out the use of doubling strategies. The opposite of such a strategy can be called a suicide strategy. It is the mathematical equivalent of making a bet at the roulette, leaving it as well as all gains on the table as long as one keeps winning, and wait until one loses for the first time. Such strategies, although admissible, do not reflect economic efficiency. More precisely we define

Definition 7. : An admissible outcome $\int_{0}^{T} H_{t} d S_{t}$ is called maximal if there is no other admissible strategy $H^{\prime}$ such that $\int_{0}^{T} H_{t}^{\prime} d S_{t} \geq \int_{0}^{T} H_{t} d S_{t}$ with $\mathbb{P}\left[\int_{0}^{T} H_{t}^{\prime} d S_{t}>\int_{0}^{T} H_{t} d S_{t}\right]>0$

In the proof of Theorem 6 , these elements play a crucial role and the heart of the proof consists in showing that every element in $K$ is dominated by a maximal element. But besides their mathematical relevance they also have a clear economic interpretation. There is no use in implementing a strategy that is not maximal as one can do better. Non-maximal elements can also be seen as bubbles, see [18].

In Theorem 6 we only assert that $S$ is a local martingale under $Q$. In fact, this technical concept cannot be avoided in this setting. Indeed, fix an $S$-integrable, predictable, admissible process $H=\left(H_{t}\right)_{0 \leq t \leq T}$ as well as a bounded, predictable, strictly positive process $\left(k_{t}\right)_{0 \leq t \leq T}$. The subsequent identity trivially holds true.

$$
\int_{0}^{t} H_{u} d S_{u}=\int_{0}^{t} \frac{H_{u}}{k_{u}} d \tilde{S}_{u}, \quad 0 \leq t \leq T,
$$

where

$$
\tilde{S}_{u}=\int_{0}^{u} k_{v} d S_{v}, \quad 0 \leq u \leq T .
$$

The message of (11) and (12) is that the class of processes obtained by taking admissible stochastic integrals on $S$ or $\tilde{S}$ simply coincide. An easy interpretation of this rather trivial fact is that the possible investment opportunities do not depend on whether stock prices are denoted in Euros or in Cents (this corresponds to taking $k_{t} \equiv 100$ above).

But it may very well happen that $\tilde{S}$ is a martingale while $S$ only is a local martingale. In fact, the concept of local martingales may even be characterized in these terms ([10, Proposition 2.5]): a semi-martingale $S$ is a local martingale if and only if there is a strictly positive, decreasing, predictable process $k$ such that $\tilde{S}$ defined in (12) is a martingale. 
Again we want to emphasize the role of the maximal elements. It turns out, see [8] and [11], that if $\int_{0}^{T} H_{t} d S_{t}$ is maximal, if and only if there is an equivalent local martingale measure $Q$ such that the process $\int_{0}^{t} H_{u} d S_{u}$ is a martingale and not just a local martingale under $Q$. One can show, see [9] and [11], that for a given sequence of maximal elements $\int_{0}^{T} H_{t}^{n} d S_{t}$, one can find one and the same equivalent local martingale measure $Q$ such that all the processes $\int_{0}^{t} H_{u}^{n} d S_{u}$ are $Q$-martingales. Another useful and related characterisation, see [8] and [11], is that if a process $V_{t}=x+\int_{0}^{t} H_{u} d S_{u}$ defines a maximal element $\int_{0}^{T} H_{u} d S_{u}$ and remains strictly positive, the whole financial market can be rewritten in terms of $V$ as a new numéraire without losing the no-arbitrage properties. The change of numéraire and the use of the maximal elements allows to introduce a numéraire invariant concept of admissibility, see [9] for details. An important result in this paper is that the sum of maximal elements is again a maximal element.

Theorem 6 above still contains one severe limitation of generality, namely the local boundedness assumption on $S$. As long as we only deal with continuous processes $S$, this requirement is, of course, satisfied. But if one also considers processes with jumps, in most applications it is natural to drop the local boundedness assumption.

The case of general semi-martingales $S$ (without any boundedness assumption) was analyzed in [10]. Things become a little trickier as the concept of local martingales has to be weakened even further: we refer to eqf04/007: Equivalent Martingale Measure and Ramifications for a discussion of the concept of sigma-martingales. This concept allows to formulate a result pertaining to a perfectly general setting.

Theorem 8. ([7, Corr. 1.2]): Let $S=\left(S_{t}\right)_{0 \leq t \leq T}$ be an $\mathbb{R}^{d}$-valued semimartingale.

There is a probability measure $Q$ on $(\Omega, \mathcal{F})$ which is equivalent to $\mathbb{P}$ and under which $S$ is a sigma-martingale if and only if $S$ satisfies the condition of no free lunch with vanishing risk with respect to admissible strategies.

One still may ask whether it is possible to formulate a version of the fundamental theorem which does not rely on the concepts of local or sigma-, but rather on "true" martingales.

This was achieved by J. Yan [38] by applying a clever change of numéraire technique, (eqf04/010: Change of Numéraire compare also [13, Section $5]$ ): let us suppose that $\left(S_{t}\right)_{0 \leq t \leq T}$ is a positive semi-martingale, which is natural if we model, e.g., prices of shares (while the previous setting of not necessarily positive price processes also allows for the modeling of forwards, futures etc.). 
Let us weaken the admissibility condition (6) above, by calling a predictable, $S$-integrable process allowable if

$$
\int_{0}^{t} H_{u} d S_{u} \geq-M\left(1+S_{t}\right) \quad \text { a.s., for } 0 \leq t \leq T .
$$

The economic idea underlying this notion is wellknown and allows for the following interpretation: an agent holding $M$ units of stock and bond may, in addition, trade in $S$ according to the trading strategy $H$ satisfying (13); she will then remain liquid during $[0, T]$.

By taking $S+1$ as new numéraire and replacing admissible by allowable trading strategies Yan obtains the following Theorem.

Theorem 9. ([38, Theorem 3.2]) Suppose that $S$ is a positive semi-martingale. There is a probability measure $Q$ on $(\Omega, \mathcal{F})$ which is equivalent to $\mathbb{P}$ and under which $S$ is a martingale if and only if $S$ satisfies the condition of no free lunch with vanishing risk with respect to allowable trading strategies.

\section{Walter Schachermayer}

\section{References}

[1] Arrow, K. (1964) The role of securities in the optimal allocation of riskbearing. Rev. Econ. Stud. 31, 91-96 (1964).

[2] Bachelier L. (1964) Théorie de la Spéculation. Ann. Sci. Ecole Norm. Sup. 17, 21-86 (1900) English translation in: The Random Character of stock market prices (P. Cootner, editor), MIT Press.

[3] Black, F, Scholes, M. (1973) The pricing of options and corporate liabilities. Journal of Political Economy 81, 637-659.

[4] Cox, J, Ross, S. (1976) The valuation of options for alternative stochastic processes. J. Financial Econom. 3, 145-166.

[5] Dalang, R.C, Morton, A, Willinger, W. (1990) Equivalent Martingale measures and no-arbitrage in stochastic securities market model. Stochastics and Stochastic Reports 29, 185-201.

[6] Delbaen, F. (1992) Representing Martingale Measures when Asset Prices are Continuous and Bounded. Mathematical Finance 2, 107-130. 
[7] Delbaen, F, Schachermayer, W. (1994) A General Version of the Fundamental Theorem of Asset Pricing. Mathematische Annalen 300, 463520 .

[8] Delbaen, F, Schachermayer, W. (1995) The no-arbitrage condition under a change of numéraire. Stochastics Stochastics Rep. 53, 213-226.

[9] Delbaen, F, Schachermayer, W. (1997) The Banach space of workable contingent claims in arbitrage theory. Ann IHP Prob Stat 33, 113-144.

[10] Delbaen, F, Schachermayer, W. (1998) The Fundamental Theorem of Asset Pricing for Unbounded Stochastic Processes. Mathematische Annalen 312, 215-250.

[11] Delbaen, F, Schachermayer, W. (2006) The Mathematics of Arbitrage. Springer Finance, 371.

[12] Duffie, D, Huang, C.F. (1986) Multiperiod security markets with differential information; martingales and resolution times. Journal of Mathematical Economics 15, 283-303.

[13] Guasoni, P, Rásonyi, M, Schachermayer, W. (2009) The Fundamental Theorem of Asset Pricing for Continuous Processes under Small Transaction Costs. Annals of Finance.

[14] Harrison, J.M, Kreps, D.M. (1979) Martingales and Arbitrage in Multiperiod Securities Markets. Journal of Economic Theory 20, 381-408.

[15] Harrison, J.M, Pliska, S.R. (1981) Martingales and Stochastic Integrals in the Theory of Continuous Trading. Stochastic Processes and their Applications 11, 215-260.

[16] Harrison, J.M, Pliska, S.R. (1983) A stochastic calculus model of continuous trading: Complete markets. Stochastic Processes and their Applications 11, 313-316.

[17] Jacod, J, Shiryaev, A.N. (1998) Local martingales and the fundamental asset pricing theorems in the discrete-time case. Finance and Stochastics (3) $2,259-273$.

[18] Jarrow, R, Protter, P, Shimbo, K. (2007) Asset price bubbles in complete markets. Advances in Math. Finance Appl. Numer. Harmon. Anal. Birkhäuser, Boston, Boston MA, 97-121. 
[19] Kabanov, Y.M. (1997) On the FTAP of Kreps-Delbaen-Schachermayer (English). Y.M. Kabanov (ed.) et al., Statistics and control of stochastic processes. The Liptser Festschrift. Papers from the Steklov seminar held in Moscow, Russia, 1995-1996. Singapore: World Scientific, 191-203.

[20] Kabanov, Y.M, Kramkov, D. (1994) No-arbitrage and equivalent martingale measures: An elementary proof of the Harrison-Pliska theorem. Theory Prob. Appl. (3) 39, 523-527.

[21] Kabanov, Y.M, Stricker, Ch. (2001) A teachers' note on no-arbitrage criteria. Séminaire de Probabilités XXXV, Springer Lecture Notes in Mathematics 1755, 149-152.

[22] Kemeny, J.G. (1955) Fair bets and inductive probabilities. Journal of Symbolic Logic (3) 20, 263-273.

[23] Kramkov, D, Schachermayer, W. (1999) The Asymptotic Elasticity of Utility Functions and Optimal Investment in Incomplete Markets. Annals of Applied Probability (3) 9, 904-950.

[24] Kreps, D.M. (1981) Arbitrage and Equilibrium in Economics with infinitely many Commodities. Journal of Mathematical Economics 8, 1535.

[25] Merton, R.C. (1973) The theory of rational option pricing. Bell J. Econ. Manag. Sci. 4, 141-183.

[26] Rogers, L.C.G. (1994) Equivalent martingale measures and no-arbitrage. Stochastics and Stochastic Reports (1-2) 51, 41-49.

[27] Ross, S. (1976) The arbitrage theory of capital asset pricing. J. Econ. Theor. 13, 341-360.

[28] Ross, S. (1977) Return, Risk and Arbitrage. Risk and Return in Finance 1, 189-218.

[29] Ross, S. (1978) A simple approach to the valuation of risky streams. J. Business 51, 453-475.

[30] Samuelson, P.A. (1965) Proof that properly anticipated prices fluctuate randomly. Industrial Management Review 6, 41-50.

[31] Schachermayer, W. (1992) A Hilbert space proof of the fundamental theorem of asset pricing in finite discrete time. Insurance: Mathematics and Economics (4) 11, 249-257. 
[32] Schachermayer, W. (1994) Martingale Measures for Discrete time Processes with Infinite Horizon. Mathematical Finance 4, 25-56.

[33] Schachermayer, W. (2005) A Note on Arbitrage and Closed Convex Cones. Mathematical Finance (1).

[34] Schachermayer, W, Teichmann, J. (2005) How close are the Option Pricing Formulas of Bachelier and Black-Merton-Scholes? Mathematical Finance (1) 18, 55-76.

[35] Shimony, A. (1955) Coherence and the Axioms of Confirmation. The Journal of Symbolic Logic 20, 1-28.

[36] Stricker, Ch. (1990) Arbitrage et Lois de Martingale. Annales de l'Institut Henri Poincaré - Probabilites et Statistiques 26, 451-460.

[37] Yan, J.A. (1980) Caractérisation d' une classe d'ensembles convexes de $L^{1}$ ou $H^{1}$. J. Azéma, M. Yor (eds.), Séminaire de Probabilités XIV, Springer Lecture Notes in Mathematics 784, 220-222.

[38] Yan, J.A. (1998) A new look at the fundamental theorem of asset pricing. J. Korean Mat. Soc. 35, 659-673.

[39] Yor M. (1978) Sous-espaces denses dans $L^{1}$ ou $H^{1}$ et représentation des martingales. C. Dellacherie et al. (eds.), Séminaire de Probabilités XII, Springer Lecture Notes in Mathematics 649, 265-309. 